\title{
Development of an Outpatient Palliative Care Protocol to Monitor Fidelity in the Emergency Medicine Palliative Care Access Trial
}

\author{
Corita R. Grudzen, MD, MSHS, Abigail M. Schmucker, ${ }^{2}$ Deborah J. Shim, ${ }^{3}$ \\ Aminat Ibikunle, MD, ${ }^{4}$ Jeanne Cho, $\mathrm{MPH}^{1}{ }^{1}$ Frank R. Chung, ${ }^{4}$ \\ and Susan E. Cohen, MD; ${ }^{5}$ and The EMPallA Outpatient Investigators ${ }^{\star}$
}

\begin{abstract}
Introduction: Palliative care is recommended for patients with life-limiting illnesses; however, there are few standardized protocols for outpatient palliative care visits. To address the paucity of data, this article aims to: (1) describe the elements of outpatient palliative care that are generalizable across clinical sites; (2) achieve consensus about standardized instruments used to assess domains within outpatient palliative care; and (3) develop a protocol and intervention checklist for palliative care clinicians to document outpatient visit elements that might not normally be recorded in the electronic heath record.

Methods: As part of a randomized control trial of nurse-led telephonic case management versus specialty, outpatient palliative care in older adults with serious life-limiting illnesses in the Emergency Department, we assessed the structural characteristics of outpatient care clinics across nine participating health care systems. In addition, direct observation of outpatient palliative care visits, consultation from content experts, and survey data were used to develop an outpatient palliative care protocol and intervention checklist.

Implementation: The protocol and checklist are being used to document the contents of each outpatient palliative care visit conducted as a part of the Emergency Medicine Palliative Care Access (EMPallA) trial. Variation across palliative care team staffing, clinic session capacity, and physical clinic model presents a challenge to standardizing the delivery of outpatient palliative care.
\end{abstract}

Keywords: advanced illness; emergency medicine; outpatient palliative care; palliative care

\section{Introduction}

\section{Background}

$\mathbf{H}$ ALF OF Older Americans visit the Emergency Department (ED) in the last month of life, making the ED a key decision point when providers establish the subsequent care trajectory. ${ }^{1}$ Palliative care interventions in the ED can both capture high-risk patients at a time of crisis and dramatically improve patient-centered outcomes. ${ }^{2,3}$ While access to inpatient palliative care consultation services is available in many hospitals nationwide, ${ }^{4}$ outpatient palliative care services are less established and there are few standard protocols for outpatient care delivery.

A study by Temel et al. demonstrated that lung cancer patients randomized to receive outpatient palliative care had greater improvements in quality of life and lower rates of depressive symptoms compared with those randomized to standard care. ${ }^{5}$ In this study, outpatient palliative care was delivered according to the National Consensus Project (NCP) for Quality Palliative Care guidelines, ${ }^{6}$ and palliative care

\footnotetext{
${ }^{1}$ Ronald O. Perelman Department of Emergency Medicine, New York University School of Medicine, New York, New York.

${ }^{2}$ Sidney Kimmel Medical College at Thomas Jefferson University, Philadelphia, Pennsylvania.

${ }^{3}$ Augusta University Medical College of Georgia, Augusta, Georgia.

${ }^{4}$ New York University School of Medicine, New York, New York.

${ }^{5}$ Department of Internal Medicine, New York University School of Medicine, New York, New York.

*For the list of investigators, see the Investigators section.

Accepted June 11, 2019.
} 
clinicians documented the care they provided accordingly; however, the visits were adapted using general guidelines and not protocolized in a way that makes them reproducible in other studies.

Two subsequent studies attempt to describe the structure of the palliative care visits in Temel et al.'s study through retrospective chart review. The first describes the components of the initial palliative care visits for 67 patients, ${ }^{7}$ and the latter examines 20 participants' palliative care and oncology visit notes to determine the key elements of an early ambulatory palliative care visit, changes over time and disease progression. ${ }^{8}$ While these analyses provide a framework for what occurs during a palliative care visit, the components captured only represent those documented in the visit note. Much of what occurs during a palliative care visit involves attending to emotion, providing methods for coping, and other nonverbal communication that may not be well documented in the physicians' notes. Additionally, these studies only assess palliative care visit components at a single outpatient clinic for patients with a specific disease type (nonsmall-cell lung cancer), so they may not be representative of outpatient palliative care visits broadly.

In response to the paucity of data on the structure and components of outpatient palliative care visits across health care systems and disease groups, a standardized outpatient palliative care protocol was developed as part of a randomized control trial of palliative care service for older adults with serious life-limiting illnesses. Here, we present the development of this outpatient palliative care protocol to facilitate reproducibility across the nine health care systems in the study, as well as to be used in future palliative care trials and outpatient palliative care practices.

\section{Objectives and hypotheses}

The objectives are to: (1) fully describe the elements of outpatient palliative care visits that are generalizable across clinical sites; (2) achieve consensus about standardized instruments used to assess domains within outpatient palliative care; and (3) develop a protocol and intervention checklist that allow palliative care clinicians to document outpatient visit elements that might not normally be recorded in the electronic heath record (EHR).

\section{Methods}

\section{Study design}

Nine health care systems participated in a randomized controlled trial in patients discharged from the ED comparing two established models of palliative care: nurse-led telephonic case management and specialty, outpatient palliative care. Eligible patients were aged 50 years or older and had advanced cancer (metastatic solid tumor) or end-stage organ failure (congestive heart failure, end-stage renal disease, or chronic obstructive pulmonary disease). Primary caregivers, defined as those aged 18 years or older and living with the patient, were also eligible to enroll.

Upon enrollment, patients were randomized to one of the intervention arms. Participants assigned to the outpatient palliative care clinic arm were scheduled for their first clinic visit within two weeks of enrollment, and subsequent visits were scheduled monthly in the outpatient setting for six months or until death. Specialty, outpatient palliative care visits are conducted in-person by a physician or nurse practitioner who is board-eligible or board-certified in hospice and palliative medicine. For the purposes of this article, we describe the protocolization of the outpatient palliative care intervention.

\section{Patient and caregiver study measures}

The primary study outcome is the change in patient's quality of life from enrollment to six months, as measured by the Functional Assessment of Cancer Therapy-General (FACT-G). The FACT-G is a validated 27-item questionnaire assessing the quality-of-life domains in physical, social, emotional, and functional well-being. ${ }^{9}$ While it has been used extensively in cancer, it is also used as an assessment of chronic illness therapy in many other serious illnesses. ${ }^{8}$

Secondary outcomes in patients include health care utilization (ED visits, hospital admissions, and hospice use) from enrollment to 12 months; loneliness, as measured by change in the Three-Item Loneliness Scale ${ }^{10}$ from enrollment to 6 months; symptom burden, as measured by change in the Edmonton Symptom Assessment Scale Revised (ESAS-r) ${ }^{11}$ from enrollment to 6 months; and quality of life, as measured by the FACT-G $\mathrm{G}^{9}$ at 3 and 12 months. Secondary outcomes in caregivers include caregiver physical and psychosocial distress, as measured by change in the Zarit Burden Interview $(\mathrm{ZBI}-12)^{12}$ from enrollment to six months; caregiver's quality of life, as measured by change in the Patient-Reported Outcome Measurement Information System (PROMIS-10) ${ }^{13}$ from enrollment to six months; and bereavement, as measured by change in the Texas Revised Inventory of Grief $(\mathrm{TRIG})^{14}$ at three months after patient death.

\section{Ethics determination}

Institutional review board approval was first obtained at the primary site, New York University School of Medicine, followed by the approval from all other study sites.

\section{Study Implementation: Challenges and Contributions}

To standardize palliative care visits across the nine health care systems participating in the Emergency Medicine Palliative Care Access (EMPallA), trial data were collected via a survey of the structural characteristics of outpatient palliative care clinics, direct observation of outpatient palliative care visits, and key informant interviews with palliative care content experts. The results were used to develop a palliative care visit protocol (see Appendix) and standard intervention checklist (Fig. 1) to monitor fidelity and to comprehensively document the contents of each palliative care visit conducted as a part of the EMPallA trial. ${ }^{15}$

\section{Assessing outpatient palliative care structural characteristics}

Outpatient palliative care directors at the nine health care systems for the EMPallA study were surveyed to assess the current state of practice. Providers participated in conference calls to verify survey responses. Results of the survey are shown in Table 1. Survey questions covered the following topics: 1) standardized palliative care assessment tools used, 2) physical and clinical outpatient model, 3) number and type 
Instructions: Document or check all that apply (what interventions did you provide during visit?)

\section{Physical}

Pharmacotherapy management offered/adjusted for pain

Pharmacotherapy for non-pain symptoms

Non-pharmacologic interventions for pain

Non-pharmacologic interventions for symptoms other than pain

Medical marijuana certification

In-office interventional procedures (such as joint injections, trigger point injections, paracentesis, thoracentesis etc.)

Referral for massage, acupuncture, physical therapy/occupational therapy

Referral for cognitive behavioral therapy, biofeedback

Other:

Psychosocial

Explored understanding of illness and treatments

Explored how patient/family like information shared

Explored hopes and worries

Educated about or encouraged mindfulness techniques

Guided imagery

Guidance on reframing, cognitive behavioral techniques

Mental health referral for patient (psychology, social work, support groups)

Mental health treatment encouraged for caregiver (psychology, social work, support groups)

Referral for counseling, support groups

$\square$ Referral for concrete home or community based services

$\square$ Referral for medico-legal partnership service or legal support

$\square$ Other:

\section{Bedside behavior and intervention}

$\square$ Supportive statements, validation/naming of emotions, non-abandonment statements

Therapeutic listening

Therapeutic touch (hand holding, hand on shoulder)

Other:

\section{Spiritual}

Exploring what brings life meaning

Exploring religious and spiritual beliefs that will inform care

Life review conversations

Contacting spiritual provider outside or within hospital to connect with patients during visits or after visits

Other:

\section{Quality/End of Life Planning}

Care Preferences discussed and documented

Advance directives forms completed: (list which forms completed)

Hospice referral

Home care referral

Other:

FIG. 1. Outpatient palliative care visit checklist.

of palliative care team members, 4) wait time for new appointments, 5) length of new patient and follow-up visits, 6) palliative care diagnoses accepted, 7) and number of clinic sessions per provider per week. Physical clinic models were classified under two categories: freestanding or embedded. A freestanding model delivers palliative care in a separate location from disease-specific care and is an independently functioning specialty clinic with their own palliative care staff. ${ }^{16}$ An embedded model involves palliative care delivery within a disease-specific clinic where visits, clinic space, and/ 


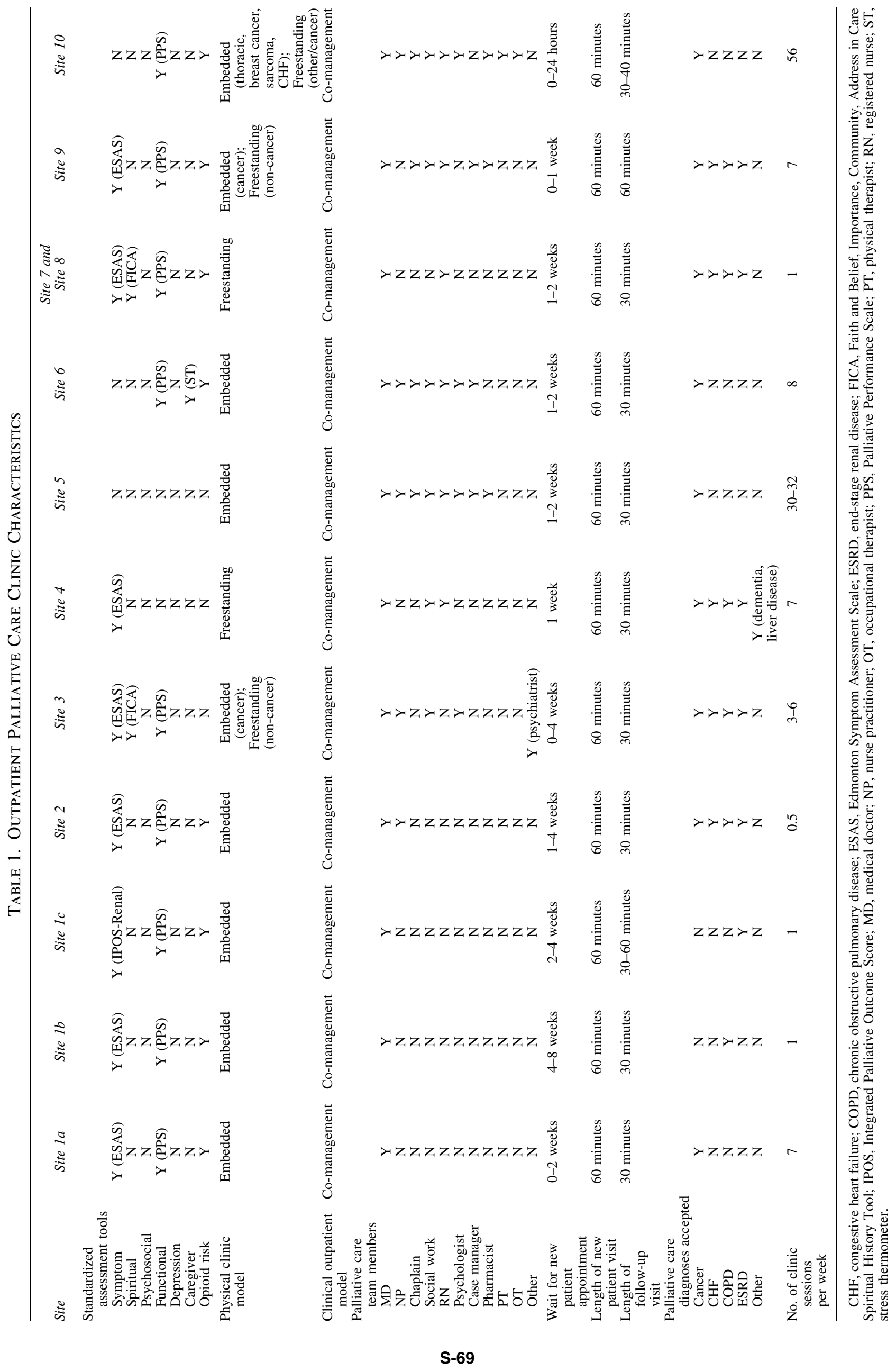


or staff are shared between disease-specific and palliative care teams. All sites were categorized under the comanagement clinical outpatient model, which involves a palliative care provider and another clinician each assuming primary responsibility for separate domains and jointly managing some patient concerns. ${ }^{17}$

\section{Direct observation of outpatient palliative care visits}

A fourth-year medical student (A.I.) observed outpatient palliative care clinic sessions at two of the participating academic medical centers. One of the clinics was a freestanding clinic and the other was embedded. Two attending palliative care physicians and several palliative care fellows were observed at each practice. The observer completed 32 total hours of observation, consisting of 8 sessions that were approximately 4 hours each. Using the NCP's eight domains of palliative care $^{6}$ as a guide, the observer recorded the frequency with which the outpatient provider addressed the domains at each visit. The observer both combined some domains and identified additional subdomains based on the observations made during the provider visits. Thematic saturation, defined as the addition of no additional subdomains, was reached before the last clinic session.

A specialty palliative care physician and content expert (S.E.C.) reviewed the notes and generalizability of the palliative care subdomains throughout the observation period. Referencing existing literature and clinical observation data, a checklist was created listing elements of a structured outpatient palliative care visit (Fig. 1).

\section{Content expert and stakeholder engagement}

The draft protocol and checklist were shared with four national specialty palliative care content experts at the annual American Academy of Hospice and Palliative Medicine (AAHPM) meeting in 2018. Outpatient palliative care directors from each of the nine health care systems then critically reviewed the outpatient protocol and checklist. After integrating national expert opinion, approval and feedback of the outpatient palliative care directors, and the specific practices of the clinical sites, the protocol and corresponding checklist were finalized. For the convenience of provider EHR documentation and data collection, the checklist was formatted into an Epic smart phrase, a predefined template that autopopulates specific text for EHR documentation.

\section{Challenges and barriers to standardizing the protocol}

Despite efforts to standardize and document the contents of an outpatient palliative care visit, several barriers still exist to delivering a uniform outpatient intervention across study sites. One barrier is the variation in the type and number of providers. Some outpatient clinics have a single palliative care physician, whereas others include palliative care team members across other disciplines, such as pharmacy and social work. Such variation affects patients' experiences with palliative care and the impact that outpatient palliative care can conceivably have on patient-centered outcomes. Additionally, the outpatient clinics differ in clinic session capacity, ranging from a half-day session every other week to
56 sessions weekly. As a result, some sites have more flexibility and ease with scheduling patients, which is also reflected in the varying wait times for new patient appointments (from 24 hours up to 4 weeks). Similarly, while many of the sites have palliative care services embedded within diseasespecific clinics, others are freestanding. This may affect the facility with which patients can engage in and attend clinic visits. Furthermore, while there is significant consensus on the standardized assessments used to evaluate patients and caregivers, they are often used disparately and present a challenge to standardizing the delivery of outpatient palliative care.

\section{Plans for dissemination and spread}

The outpatient visit protocol and intervention checklist developed in this study are integrated into Epic as a smart phrase and thus broadly accessible for clinical and research applications across health care systems globally. For instance, the intervention checklist template could be used clinically to guide a newly opened outpatient palliative care clinic or to standardize outpatient palliative care in future pragmatic trials without causing undue burden to existing palliative care providers. Additionally, we plan to conduct future analyses to measure the impact of outpatient clinic characteristics, such as the number of sessions per week and freestanding versus embedded clinic model, on patient and caregiver outcomes.

\section{Acknowledgments}

This work was (partially) supported through a PatientCentered Outcomes Research Institute (PCORI) Award (PLC-1609-36306) and a Medical Student Training in Aging Research (MSTAR) grant (1T35AG050998-01) from the National Institute on Aging (A.M.S.).

\section{The EMPallA Outpatient Investigators}

Nancy E. Bael, MD, NYU Langone Health; Paige C. Barker, MD, University of Florida Health; Jeffery T. Berger, NYU Langone Health; Angela Chmielewski, MD, Beaumont Hospital; Ellin Gafford, MD, Ohio State University Wexner Medical Center; Jennifer Kapo, MD, Yale School of Medicine; Arum Kim, MD, FAAPMR, NYU Langone Health; Joshua R. Lakin, MD, Brigham and Women's Hospital; Joseph Lowy, MD, NYU Langone Hospital; Jennifer Scherer, MD, NYU Langone Health.

\section{Author Disclosure Statement}

No competing financial interests exist.

All statements in this report, including its findings and conclusions, are solely those of the authors and do not necessarily represent the view of the Patient-Centered Outcomes Research Institute (PCORI), its Board of Governors, or Methodology Committee.

\section{Supplementary Material}

Supplementary Appendix 


\section{References}

1. Smith AK, McCarthy E, Weber E, et al.: Half of older Americans seen in emergency department in last month of life; most admitted to hospital, and many die there. Health Aff (Millwood) 2012;31:1277-1285.

2. Wu FM, Newman JM, Lasher A, Brody AA: Effects of initiating palliative care consultation in the emergency department on inpatient length of stay. J Palliat Med 2013; 16:1362-1367.

3. Grudzen CR, Richardson LD, Johnson PN, et al.: Emergency department-initiated palliative care in advanced cancer a randomized clinical trial. JAMA Oncol 2016;2: 591-598.

4. Weissman DE, Meier DE: Operational features for hospital palliative care programs: Consensus recommendations. J Palliat Med 2008;11:1189-1194.

5. Temel JS, Greer JA, Muzikansky A, et al.: Early palliative care for patients with metastatic non-small-cell lung cancer. N Engl J Med 2010;363:733-742.

6. National Consensus Project for Quality Palliative Care: Clinical Practice Guidelines for quality palliative care, executive summary. J Palliat Med 2004;7:611-627.

7. Jacobsen J, Jackson V, Dahlin C, et al.: Components of early outpatient palliative care consultation in patients with metastatic nonsmall cell lung cancer. J Palliat Med 2011; 14:459-464.

8. Yoong J, Park ER, Greer JA, et al.: Early palliative care in advanced lung cancer: A qualitative study. JAMA Intern Med 2013;173:283-290.

9. Cella DF, Tulsky DS, Gray G, et al.: The Functional Assessment of Cancer Therapy scale: Development and validation of the general measure. J Clin Oncol 1993;11:570 579.

10. Russell D, Peplau LA, Cutrona CE: The revised UCLA Loneliness Scale: Concurrent and discriminant validity evidence. J Pers Soc Psychol 1980;39:472-480.
11. Chang VT, Hwang SS, Feuerman M: Validation of the Edmonton Symptom Assessment Scale. Cancer 2000;88: 2164-2171.

12. Bedard M, Molloy DW, Squire L, et al.: The Zarit Burden Interview: A new short version and screening version. Gerontologist 2001;41:652-657.

13. Hays RD, Bjorner JB, Revicki DA, et al.: Development of physical and mental health summary scores from the patient-reported outcomes measurement information system (PROMIS) global items. Qual Life Res 2009;18:873880.

14. Faschingbauer TR, Devaul RA, Zisook S: Development of the Texas Inventory of Grief. Am J Psychiatry 1977;134: 696-698.

15. Grudzen CR, Shim DJ, Schmucker AM, et al.: Emergency Medicine Palliative Care Access (EMPallA): Protocol for a multicentre randomised controlled trial comparing the effectiveness of specialty outpatient versus nurse-led telephonic palliative care of older adults with advanced illness. BMJ Open 2019;9:e025692.

16. Finlay E, Rabow MW, Buss MK: Filling the gap: Creating an outpatient palliative care program in your institution. Am Soc Clin Oncol Educ book Am Soc Clin Oncol Annu Meet 2018;111-121.

17. Smith AK, Thai JN, Bakitas MA, et al.: The diverse landscape of palliative care clinics. J Palliat Med 2013;16: 661-668.

Address correspondence to: Corita R. Grudzen, MD, MSHS Ronald O. Perelman Department of Emergency Medicine New York University School of Medicine 227 E 30th Street New York, NY 10016

E-mail: Corita.grudzen@nyulangone.org 